\title{
Changing Fear: The Neurocircuitry of Emotion Regulation
}

\author{
Catherine A Hartley ${ }^{1}$ and Elizabeth A Phelps ${ }^{\star, 2}$ \\ ${ }^{1}$ Department of Psychology, New York University, New York, NY, USA; ${ }^{2}$ Departments of Psychology and Neural Science, \\ New York University, New York, NY, USA
}

\begin{abstract}
The ability to alter emotional responses as circumstances change is a critical component of normal adaptive behavior and is often impaired in psychological disorders. In this review, we discuss four emotional regulation techniques that have been investigated as means to control fear: extinction, cognitive regulation, active coping, and reconsolidation. For each technique, we review what is known about the underlying neural systems, combining findings from animal models and human neuroscience. The current evidence suggests that these different means of regulating fear depend on both overlapping and distinct components of a fear circuitry.

Neuropsychopharmacology Reviews (2010) 35, 136-146; doi: 10.1 038/npp.2009.121; published online 26 August 2009
\end{abstract}

Keywords: emotion regulation; fear conditioning; extinction; amygdala

\section{INTRODUCTION}

The ability to respond emotionally to salient cues in our environment is critical for adaptive human function. Emotional responses can sharpen our perceptual faculties, facilitate rapid action and decision making, foster social connections, and enhance our recollection of important events (see Phelps and LeDoux, 2005 for a review). However, our ability to modify or control the nature of our emotional responses as circumstances change is equally important. The term 'emotion regulation' refers to the different types of regulatory processes that can control the physiological, behavioral, and experiential components of our affective responses (Gross and Thompson, 2007). These include automatic forms of regulation that flexibly alter our emotional responses as we learn about changing stimulusoutcome contingencies in our environment, as well as intentionally deployed techniques. We may change how we think about an emotion-evoking stimulus, or shift our focus of attention to diminish an undesired emotion. We may also take action to avoid or cope with a distressing situation or to bring about a positive outcome. Effective regulation of emotion through these various processes is essential for both our mental and physical well being.

${ }^{*}$ Correspondence: Dr E Phelps, Departments of Psychology and Neural Science, New York University, NYU Medical Center, 6 Wash PI, 863, New York, NY 10003, USA, Tel: +1 212998 8337, Fax: +1212 995 4349, E-mail: liz.phelps@nyu.edu

Received 19 May 2009; revised 16 July 2009; accepted 20 July 2009
In this review, we examine the functional architecture underlying the regulation of fear, focusing on four different types of regulatory processes: extinction, cognitive emotion regulation, active coping, and reconsolidation. During extinction, fear is diminished through learning that a previously threatening stimulus no longer signals danger. Cognitive emotion regulation involves using various mental strategies to modify a fear response. In active coping, fear is regulated through the performance of behaviors that reduce exposure to a fear-evoking stimulus. Finally, a fear memory can be disrupted after it is recalled through pharmacological or behavioral manipulations that block its reconsolidation. Our understanding of the neurocircuitry underlying the control of fear stems from research across species clarifying the mechanisms by which we learn and modify emotional associations, as well as studies exploring forms of cognitive emotion regulation that are uniquely human. In each section, we first review what is known about the neurocircuitry of the regulatory method from the non-human animal literature, followed by a review of the evidence available from human studies. We also briefly highlight the relevance of each regulatory method to the treatment of fear-related anxiety disorders.

Failure to properly regulate fear responses has been associated with various forms of psychopathology. For example, some forms of anxiety disorders are thought to involve dysfunction in the neural systems underlying the extinction of fear learning (see Rauch et al, 2006) and are treated with extinction-based exposure therapies. Facilitating emotion regulation through intentional cognitive 
mechanisms is a primary aim of cognitive-behavioral psychotherapy, a successful approach to the treatment of depression and other psychological disorders. Active coping may be used to attenuate learned fear responses and mitigate the functional impairments engendered by a fearevoking stimulus. Finally, reconsolidation may permit the permanent modification of the pathological traumatic memories. An improved understanding of the neurocircuitry of normal emotion regulation sheds light on the potential mechanisms underlying psychiatric disorders, and may aid in the development of more effective treatments for these conditions.

\section{EXTINCTION}

A primary technique for investigating the mechanisms underlying emotional associative learning is Pavlovian conditioning. During a typical Pavlovian fear conditioning paradigm, a previously neutral stimulus, such as a tone (the conditioned stimulus, or CS) acquires emotional significance through pairing with an aversive stimulus, such as a footshock (the unconditioned stimulus or US). The footshock elicits a range of automatic, unconditioned fear responses, such as freezing and increased heart rate or blood pressure. After a few tone-shock pairings, the presentation of the CS alone is capable of eliciting a conditioned fear response (CR). In the real world, such stimulus-reinforcer associations support our ability to identify and avoid potential environmental dangers.

Extinction refers to the gradual decrease in the expression of the CR that occurs when the CS is presented repeatedly without the reinforcement of the US. Extinction learning involves the formation of a novel stimulus-outcome association. The CS that earlier predicted danger now predicts safety. This new extinction memory does not erase or overwrite the memory for the original CS-US association. This is evidenced by the re-emergence of the CR in certain circumstances including a shift in context (renewal), unsignaled presentation of the US (reinstatement), or the mere passage of time (spontaneous recovery) (see Bouton, 2004 for a review).

Studies investigating the neural mechanisms of fear conditioning across species indicate that the amygdala has a critical function in the acquisition, storage, and expression of conditioned fear. The lateral nucleus (LA) of the amygdala is thought to encode the association between CSand US-related sensory inputs. When the CS is present, the LA excites the central nucleus (CE), which controls passive forms of expression of the $\mathrm{CR}$ through descending projections to the brainstem and hypothalamus. The LA also has indirect projections to the CE, through the basal nucleus (B) and the intercalated (ITC) cell masses, clusters of inhibitory GABAergic neurons. The B itself also projects directly to the ITC. These pathways provide multiple potential circuits for gating fear expression. Knowledge of the fear conditioning circuitry has allowed researchers to investigate functional changes that occur during extinction.

Research in rats using lesions, pharmacological manipulations, and electrophysiology are providing an increasingly detailed model of the neural circuitry of fear extinction. Recent studies in human beings have been consistent with the animal literature. This body of research suggests that interaction between the amygdala, the ventromedial prefrontal cortex (vmPFC), and the hippocampus supports the acquisition, storage, retrieval, and contextual modulation of fear extinction (see Sotres-Bayon et al, 2006; Quirk and Mueller, 2008 for a review).

As the amygdala is necessary for the expression of conditioned fear (for reviews, see LeDoux, 2000; Maren, 2001), lesions are not typically used to investigate the function of the amygdala in fear extinction. However, pharmacological and electrophysiological studies in rats suggest that the amygdala has an important function in the acquisition and consolidation of fear extinction. Recent studies have shown that blockade of NMDA (Sotres-Bayon et al, 2007) or glutamate (Kim et al, 2007) receptors within the basolateral amygdala complex (BLA) impaired extinction learning and that the blockade of mitogen-activated protein kinase (MAPk) activity in the basolateral nucleus entirely prevented the acquisition of extinction (Herry et al, 2006). Furthermore, several studies suggest that morphological changes in the BLA synapses after extinction training support the consolidation of extinction learning (Lin et al, 2003; Chhatwal et al, 2005, 2006; Markram et al, 2007). Consistent with the idea that extinction constitutes new learning, not erasure of the original fear memory, Repa et al (2001) identified a population of neurons in the lateral amygdala whose response to the CS decreased during extinction training, as well as a second population in which the CS response remained high despite a decrease in the expression of conditioned fear. This finding provides further evidence that the amygdala supports the maintenance of the original fear memory when simultaneously facilitating extinction learning.

Although the amygdala seems to be critical for the acquisition of extinction learning, convergent evidence suggests that the vmPFC is necessary for the retention and recall of extinction. In line with the well-documented observation in human beings and primates that damage to the PFC leads to perseverative behavior (see Sotres-Bayon et al, 2006 for a review), Morgan et al (1993) observed that rats with vmPFC lesions required many more unreinforced presentations of the CS to extinguish conditioned responding. A subsequent study pointed to the infralimbic (IL) region of the vmPFC as a potential site of extinction consolidation, reporting that pre-training IL lesions left within-session acquisition of extinction intact, but impaired extinction retrieval on the following day (Quirk et al, 2000). Infusion studies showing that disruption of protein synthesis (Santini et al, 2004), MAPk blockade (Hugues et al, 2006), and administration of an NMDA antagonist (Burgos-Robles et al, 2007) within the vmPFC all 
impair retrieval of extinction suggest that the plasticity in this region supports extinction consolidation. Electrophysiological evidence suggests that the IL inhibits the expression of conditioned fear during extinction through reciprocal connections with the amygdala. IL neurons show increased activity to the CS during extinction retrieval (Milad and Quirk, 2002). Stimulation of IL neurons both decreases the responsiveness of CE neurons (Quirk et al, 2003) and diminishes conditioned responding to a nonextinguished CS (Milad et al, 2004). Furthermore, the degree of high-frequency bursting activity in IL neurons after extinction was correlated with recall of extinction on the following day (Burgos-Robles et al, 2007). Two current theories propose that inhibition of fear expression during extinction may occur through IL activation of inhibitory interneurons in the LA (Rosenkranz et al, 2003) or through IL activation of the inhibitory ITC projections to the CE (Paré et al, 2004).

After extinction, contextual information has a critical function in determining whether the original fear memory or the new extinction memory should control fear expression (see Bouton, 2004 for a review). Evidence suggests that hippocampal projections to the vmPFC and the amygdala mediate the context-dependent expression of extinction (Fanselow, 2000; Ji and Maren, 2005). Rats with hippocampal lesions show impaired contextual reinstatement of the CR after unsignaled US presentations (Wilson et al, 1995). Inactivation of the hippocampus after extinction learning prevents the renewal of conditioned fear in a non-extinction context (Corcoran and Maren, 2001, 2004; Hobin et al, 2006). Furthermore, inactivation of the hippocampus before extinction learning impairs extinction recall on the subsequent day (Corcoran et al, 2005), suggesting that the hippocampus regulates fear expression both outside and within the extinction context. One suggestion is that the hippocampus controls the context-specific retrieval of extinction through projections to the vmPFC (Corcoran and Quirk, 2007). Another possibility is that the hippocampus gates fear expression directly through projections to the LA. A recent study showed that the context-specific modulation of both fear expression and CS-evoked activity in the LA (greater responding in the non-extinction context than in the extinction context) depends on the hippocampus (Maren and Hobin, 2007). Although clarification of the precise circuitry requires further investigation, there is strong evidence that the hippocampus, through communication with the vmPFC and the amygdala, regulates the contextual modulation of fear expression during extinction retrieval.

Research examining extinction learning in humans has been largely consistent with the findings from rodent studies. Initial fMRI studies of extinction learning in humans reported an increase in amygdala activation during early extinction after the shift in the CS-US contingency (LaBar et al, 1998; Gottfried and Dolan, 2004; Knight et al, 2004; Phelps et al, 2004). Amygdala activation decreased as extinction progressed and remained low during extinction retrieval. The magnitude of the decrease in amygdala activation during extinction learning correlated with the degree of participants' extinction retrieval (Phelps et al, 2004). These findings further support a function for the amygdala in extinction learning and confirm that amygdala activity is reduced during extinction retrieval.

The first fMRI study examining both extinction learning and subsequent retrieval in humans supported the function of the vmPFC in extinction retrieval (Phelps et al, 2004). Fear conditioning fMRI studies have typically reported decreases in the blood oxygen level-dependent (BOLD) signal in the vmPFC below the resting baseline when the CS is presented during fear acquisition (Gottfried and Dolan, 2004; Phelps et al, 2004). BOLD signal in this region increases toward baseline during initial extinction learning, and increases further during extinction retrieval (Phelps et al, 2004). A similar subgenual anterior cingulate region has been proposed as a potential homologue of the rodent IL region (Kim et al, 2003), and may inhibit conditioned fear expression during extinction retrieval, as suggested by the animal literature. Subsequent studies have also observed increased vmPFC activation during extinction retrieval (Kalisch et al, 2006; Milad et al, 2007). Furthermore, the degree of increase in activity in the vmPFC as well as the thickness of the cortex in this region are correlated with the degree of extinction success during recall (Milad et al, 2005, 2007).

FMRI studies examining the context-dependent recall of extinction in humans point to an important function for the hippocampus (Kalisch et al, 2006; Milad et al, 2007). In such studies, context is manipulated through changes in the background color or the visual scene in which the CS is presented. Extinction learning takes place only in one of the contexts, allowing activation correlated with contextdependent retrieval to be examined. Increased hippocampal activation was observed during extinction retrieval (Kalisch et al, 2006; Milad et al, 2007). Furthermore, activation in the hippocampus was positively correlated with vmPFC activation, providing further support for the notion that the hippocampus may mediate context-dependent extinction recall through connections with the vmPFC. Finally, confirming the function of the hippocampus in contextual reinstatement, a human lesion study found that contextual reinstatement of the $\mathrm{CR}$ was impaired in individuals with hippocampal lesions (LaBar and Phelps, 2005), similar to the findings observed in rodents (Wilson et al, 1995).

Consistent with studies in animal models, extinction learning in humans seems to depend on the integrated functioning of a neural circuit that includes the amygdala, the vmPFC, and the hippocampus. This convergent evidence across species suggests that the neural mechanisms supporting fear extinction are highly phylogenetically conserved.

Although fear learning is essential for survival, stimulusoutcome associations learned through Pavlovian conditioning may give rise to clinical psychopathology when reactivity to the CS persists in the absence of a CS-US contingency. Knowledge of the neural circuitry of fear 
extinction has informed behavioral and pharmacological approaches for the treatment of anxiety disorders, and may aid in the identification of individuals who might be at risk of developing these disorders. Extinction-based exposure therapies have been widely explored as potential avenues of treatment for anxiety disorders (Barlow, 2002; Garakani et al, 2006). Posttraumatic stress disorder (PTSD), in which patients display spontaneous or cue induced re-experiencing of a traumatic event, may result from failure to consolidate and retrieve extinction learning (see Rauch et al, 2006 for a review). Consistent with this theory, PTSD patients exhibit deficits in extinction retention (Orr et al, 2000 ), along with reduced vmPFC and hippocampal volume and activity and increased amygdala activity (Gilbertson et al, 2002; Bremner, 2006; Liberzon and Martis, 2006; Shin et al, 2006). In addition, PTSD symptoms may improve after exposure therapy (Rothbaum and Schwartz, 2002; Foa, 2006). Research showing the facilitation of extinction learning through pharmacological means (Walker et al, 2002; Ressler et al, 2004; see Anderson and Insel, 2006; Quirk and Mueller, 2008 for reviews) suggests that drug administration may enhance the efficacy of such extinctionbased therapies. Finally, a recent study reported that mice lacking the serotonin transporter gene show marked deficits in extinction retention (Wellman et al, 2007). As human beings with the low-expressing short allele variant of this gene exhibit decreased functional connectivity between the vmPFC and amygdala (Pezawas et al, 2005), this suggests a possible genetic basis for individual differences in extinction learning, as well as a potential risk factor for the development of anxiety disorders.

\section{COGNITIVE REGULATION STRATEGIES}

Although it is likely that some other primates have the cognitive capability to influence emotion (Barnes et al, 2008), the use of cognitive strategies to control emotion has been studied exclusively in human beings. Humans regularly use thoughts to alter emotions. Whether it is choosing to reinterpret the significance of an event or deciding to focus attention on the less fearful aspects of a situation, we tune our cognition in the service of generating more adaptive emotional and social reactions. Many different cognitive strategies can be used to regulate emotion, including reappraisal, selective attention, and suppression (see Gross, 1998 for a review). Although some forms may be automatically recruited (see Mauss et al, 2007 for a review), cognitive regulation techniques often require the active engagement of the participants in an effort to alter the emotional response through changing the appraisal of the significance of events or engagement with the event. Cognitive regulation strategies can be taught and practiced, as in cognitive therapy. They can also become habitual and easier to enact over time.

Studies outlining the neural systems mediating the cognitive regulation of emotion have examined a range of strategies. One of the earlier studies examined how a cognitive strategy can increase the expression of fear or negative affect (Phelps et al, 2001). This study used an Instructed Fear paradigm in which participants are verbally instructed that they might receive a shock paired with one stimulus (threat), but not another (safe). Although a shock was never delivered, simply anticipating a potential shock with the threat stimulus led to increased arousal and increased activation of the left amygdala (Phelps et al, 2001). It was also shown that damage to the left, but not right, amygdala resulted in an impaired expression of instructed fear (Funayama et al, 2001). This hemispherespecific effect may be due to the fact that the potential threat is communicated symbolically through language, which typically has a left hemisphere representation. The laterality of instructed fear is in contrast to fear conditioning, which is impaired after lesions of either the right or left amygdala (LaBar et al, 1995). Unlike fear conditioning, it is unlikely that the symbolic, cognitive representation generated in instructed fear relies on the amygdala for the formation or storage of the fear representation, but nevertheless the amygdala has a critical function in the expression of this cognitive means of fear learning. These findings suggest that even though human beings have developed complex cognitive and social means for acquiring and representing fear, they take advantage of phylogenetically shared mechanisms for fear expression (see Olsson and Phelps, 2007).

The majority of the studies on the cognitive regulation of emotion examine means to diminish fear or negative affect. Although a few cognitive techniques have been investigated to diminish fear, most studies examine strategies that emphasize reinterpreting the emotional significance of the event (see Ochsner and Gross, 2008 for a review). Theories of emotion highlight how our interpretation or appraisal of an event can have a critical function in our emotional response (Scherer, 2005). By manipulating this appraisal process, one can alter the emotional response. A study by Ochsner et al (2002) used a reappraisal strategy in which participants were presented with negative emotional scenes and asked to reinterpret the events depicted in the scene to reduce their negative affective response. When using this strategy, a participant might imagine when a scene depicts a bloody wound that it is fake or the wound is less painful than it appears. Reappraisal has been shown to be effective at reducing negative effect using both self-report and physiological measures of emotion (Ochsner and Gross, 2008). An examination of the patterns of brain activation in this initial study revealed that the reappraisal of negative scenes, as opposed to just attending to them, resulted in increased activation of both dorsolateral PFC (DLPFC) and ventrolateral PFC (VLPFC) regions along with dorsal anterior cingulate, and decreased activation of a region of the orbitofrontal cortex and the amygdala (Ochsner et al, 2002). It was proposed that underlying the reappraisal of negative effect, the engagement of the DLPFC may be linked to executive control processes required in the online 
manipulation of the interpretation of scenes, and the decrease of amygdala activation may reflect the cognitive control of subcortical mechanisms linked to the representation of negative emotional value (Ochsner et al, 2002).

On account of this initial report (see also Beauregard et al, 2001), a number of studies have examined the reinterpretation of negative effect. These studies consistently report decreased amygdala activation and increased activation of the DLPFC and/or VLPFC, along with some involvement of medial PFC (mPFC) regions. However, across studies, the precise location and/or laterality of these PFC regions vary, perhaps because of subtle difference in the stimuli or strategy used (see Ochsner and Gross, 2008 for a review). In spite of these differences, a general model of the cognitive regulation of fear or negative affect has emerged. In this model, the DLPFC (eg, Ochsner et al, 2002; Delgado et al, 2008) is involved in the effortful manipulation or interpretation of the stimulus and the VLPFC may have a function in the selection of emotion interpretation (eg, Wager et al, 2008). The changes observed in the amygdala are the result of the top down modulation of the emotional meaning of the stimulus. One important aspect of this model is that the DLPFC does not project directly to the amygdala (McDonald et al, 1996; Barbas, 2000). Instead its influence on the amygdala is likely mediated by ventral and mPFC regions that have stronger connections with the amygdala (Urry et al, 2006). Although this model of emotion regulation is somewhat speculative given the inconsistency of precise PFC regions observed across studies (see Ochsner and Gross, 2008) and the dependence on a single, correlational neuroscience technique, it provides a working neural framework on which to build when trying to understand the cognitive regulation of fear.

A critical aspect of the studies of cognitive regulation of fear is that a decrease in the amygdala response is typically associated with an increased response in the PFC, suggesting a possible inhibitory relationship. Much like extinction, it is suggested that this PFC inhibition is critical to the control of fear. In an effort to directly compare the function of the PFC in the inhibition of the amygdala across extinction and cognitive regulation, Delgado et al (2008) examined the regulation of conditioned fear. In this study, the CSs were colored squares and fear was assessed using a physiological measure. When instructed to regulate, participants used a strategy in which they generated an image of a soothing nature scene containing the color of the square. In contrast to attending trials, using this cognitive strategy resulted in a decrease in the CR. This decrease in fear was accompanied by increased activation of the DLPFC, decreased activation of the amygdala, and increased activation of a region of the vmPFC overlapping with that observed in a similar study on fear extinction (Phelps et al, 2004; Delgado et al, 2008). In a direct comparison with data from the extinction study, similar patterns of activation were observed in the amygdala and vmPFC when fear was diminished through either extinction or cognitive regulation, although only the regulation study reported increased activation of the DLPFC, consistent with a function for this region in the online manipulation or reinterpretation of the meaning of the CS. When comparing responses across these regions, it was found that responses in the vmPFC were correlated with those observed in both the DLPFC and the amygdala. These results suggest a model by which the DLPFC inhibition of the amygdala during cognitive regulation is mediated through the same vmPFC region thought to mediate the inhibition of fear with extinction. It is possible that much like the generation of fear through cognitive means relies on the amygdala for expression, the inhibition of fear through cognitive means relies on a phylogentically shared vmPFC-amygdala circuitry. Although most cognitive regulation techniques are unique to humans, by linking components of the neural circuitry of extinction with regulation, we gain some insight into additional potential details of the neural mechanisms underlying the cognitive control of fear.

Laboratory studies of cognitive emotion regulation techniques that alter the appraisal of events to change emotion are proposed to mirror the processes used during cognitive therapy in the clinic. For example, individuals with depression often exhibit biases toward negative appraisals of events. A primary goal of cognitive therapy is to enable the patient to form more realistic evidencebased appraisals of a situation, thereby regulating the associated emotional responses (Allen et al, 2008). This training likely uses the neural pathways engaged during cognitive regulation. Consistent with this suggestion, a recent study reported that fMRI activation in response to fearful faces in the amygdala and subgenual ACC, a subregion of the vmPFC, predicts success of cognitivebehavioral therapy treatment in PTSD patients (Bryant $e t$ al, 2007). This suggests that the efficacy of such treatment may rely on the functional integrity of the earlier discussed neural circuitry and the success with which individuals are able to engage these regulatory mechanisms.

\section{ACTIVE COPING}

If one reflects on the means used to regulate emotions outside the laboratory, active coping might emerge as the most common technique. We routinely choose to engage in actions that result in positive emotional outcomes and avoid those that have negative consequences. Given the frequency of emotionally adaptive actions, it is somewhat surprising how little research has been conducted exploring the mechanisms of regulating emotion, specifically fear, through action. One potential reason is that learning an action to avoid a fearful event requires complex learning processes. First one needs to learn that the situation or event is linked to an aversive consequence, which is essentially Pavlovian fear conditioning. As outlined above, the mechanisms outlining Pavlovian fear conditioning have been extensively investigated. However, active coping involves an additional, second instrumental learning 
process, which is using this knowledge to reinforce an action that then diminishes the fear response. Although there have been some studies exploring this additional, second instrumental learning process, our knowledge of the function of fear in guiding instrumental actions is not nearly as detailed as our understanding of Pavlovian fear learning. However, we can use our knowledge of fear conditioning as a starting point for understanding the mechanisms underlying active coping.

To briefly review (also see the section on Extinction), the amygdala has a critical function in the acquisition, storage, and expression of conditioned fear. Within the amygdala, the LA is thought to encode and store the association between CS and US. The LA projects to the CE, which, through descending projections to the brainstem and hypothalamus, controls the passive expression of conditioned fear. The LA also projects to the $\mathrm{B}$, which projects to the nucleus accumbens (NA) in the striatum. The striatum is often not only cited for its function in decision making and reward learning (Rangel et al, 2008), but it is also proposed to be a region in which action and motivation are integrated more broadly (Morgenson et al, 1980). This B to NA pathway is suggested to enable active coping (LeDoux and Gorman, 2001).

Evidence in support of this proposed function of B in mediating active coping is derived from a study examining the escape from fear (EFF) paradigm. An advantage of this paradigm is that it separates the Pavlovian and instrumental learning components of active coping. In EFF, the rat first undergoes fear conditioning. In a second stage, the rat is given the option to take an action (cross the chamber) to terminate the CS, thus reducing exposure to the feareliciting event. In this paradigm, the termination of the CS becomes a conditioned reinforcer for the instrumental action. An investigation by Amorapanth et al (2000) found that diminishing fear through active coping relied on a circuitry within the amygdala that can be dissociated from the standard means of expression of conditioned fear. By placing lesions in the different amygdala subnuclei, it was found that damage to the LA, the proposed site of the CS-US representation, impairs both freezing to the CS and the ability to take an action to avoid the CS. Rats with lesions confined to the $\mathrm{CE}$ were able to learn an action to terminate the CS, even though they failed to show the typical expression (ie freezing) of conditioned fear. In contrast, damage to $B$ resulted in the opposite pattern of results, that is failure to learn an action to terminate the CS, but normal expression of the CR. On the basis of these results, it is suggested that the LA stores the CS-US connection and mediates active coping responses through its projections to the $\mathrm{B}$, which then projects to the striatum to convey the reinforcing nature of the instrumental action (see LeDoux and Gorman, 2001).

Although there is no evidence yet from animal models that specifically investigate the striatum's function in active coping, data from a recent human imaging study by Delgado et al (in press) was consistent with the proposed pathway implicating amygdala-striatal interactions in a fear avoidance learning. In this with study, subjects were taught that when they were presented a CS that predicted shock, they could 'turn-off' the delivery of the shock by learning the appropriate action. When the shock was avoided, less conditioned fear and amygdala activation was observed, but learning the active coping response resulted greater in activation of the striatum. These results are consistent with a function for the striatum in integrating motivation and action in avoidance learning.

Several other studies have also implicated the amygdala, particularly the LA and B, in mediating active coping (eg, Gabriel et al, 2003; see Cain and LeDoux, 2008 for a review). In addition to engaging an overlapping, but different neural circuitry than Pavlovian fear conditioning, research on EFF and other active coping paradigms highlight a few other factors concerning the relation between these two fear regulation paradigms that are important to note as we consider the translation of active coping techniques to clinical practice. One factor is the relation between the learning and expression of Pavlovian and instrumental fear responses. Although Pavlovian conditioning can assist in learning the instrumental coping response by creating the fear that is then terminated, a CR can also inhibit action making it harder to learn an avoidance action. This was exemplified in a study by Choi and LeDoux (2003) in which rats had to learn to shuttle between the two sides of a chamber to avoid a shock at the end of a CS presentation. Consistent with Amorapanth et al (2000), lesions of the B or LA impaired retention on this task and lesions of CE had no impact for rats that learned the avoidance response successfully. However, CE lesions enhanced or rescued learning in the subset of rats that had difficulty in learning the avoidance response because of excessive freezing. By damaging the $\mathrm{CE}$ and the defensive freezing response, these high-fear rats were more likely to discover the correct avoidance action. This study highlights the variable nature of fear and how excessive fears may make it harder to learn how to cope.

A second factor that may be relevant in the potential application of active coping techniques in the clinic is the potential for the return of fear. As mentioned above (see section on Extinction), when a conditioned fear undergoes extinction, it may return in a number of circumstances. A potentially important advantage of learning an instrumental response to cope with fear is a reduction in the chance of fear returning. Using an EFF paradigm, Cain and LeDoux (2007) reported that learning an active coping response not only reduced fear to a CS, but also that this reduction of fear lasted for an extended period of time. Unlike traditional extinction, there was no evidence of spontaneous recovery of fear after EFF training. Although research on this topic is limited, this initial behavioral result suggests a potentially important advantage to learning to cope with fears as opposed to simply extinguishing them.

Although the clinical treatment of mood and anxiety disorders has focused predominantly on extinction-based 
exposure therapies or cognitive regulation techniques, research on active coping suggests an alternative approach to overcoming anxiety. In the laboratory, the performance of active avoidance behaviors or the engagement of coping strategies that reduce exposure to a fear-evoking stimulus has been shown to reduce the physiological and behavioral expression of conditioned fear. Symptoms such as hyperarousal and physical immobility that are commonly observed in individuals with PTSD may reflect a failure or inability to initiate such active coping behaviors. Consistent with this hypothesis, individuals with PTSD exhibit reduced activity in the striatum (Lanius et al, 2001; Lindauer et al, 2004), a region thought to be critical for the establishment of coping responses. The development of such actionoriented approaches has been advocated as a potentially powerful but underused mechanism for the treatment of conditions such as PTSD (van der Kolk, 2006).

\section{RECONSOLIDATION}

A relatively new and exciting technique being investigated as a means to regulate emotion is the blockade of reconsolidation. The classic view of memory suggests that immediately after learning, there is a period of time during which the memory is fragile and labile, but after sufficient time has passed the memory is, more or less, permanent. During this consolidation period, it is possible to disrupt the formation of the memory, but once this time window has passed, the memory may be modified or inhibited, but not eliminated. However, recent studies support an alternative view of memory in which every time a memory is retrieved, the underlying memory trace is once again labile and fragile, requiring another consolidation period called reconsolidation. This reconsolidation period allows another opportunity to disrupt the memory. Given that fear memories can, at times, be maladaptive, contributing to fear or anxiety disorders, the possibility of disrupting an earlier acquired fear memory by blocking reconsolidation could have significant clinical implications.

The recent interest in reconsolidation was sparked by a finding by Nader et al (2000), showing that conditioned fear can be eliminated by blocking reconsolidation. In this study, rats were conditioned to fear a tone CS. After consolidation, some of the rats were presented with an unreinforced presentation of the CS, which served to reactivate the fear memory trace. This reactivation was followed immediately by an injection of either a protein synthesis inhibitor (anisomycin) or a saline solution into the LA nucleus of the amygdala. Even though the fear memories were fully consolidated, the rats that received anisomycin after reactivation failed to show evidence of conditioned fear, indicating that protein synthesis is necessary for the successful re-storage of memories during reconsolidation. The rats that received either a saline injection after reactivation of the CS or anisomycin without reactivation showed normal conditioned fear. These results suggest that fear memories undergo reconsolidation when they are retrieved and that this reconsolidation process can be disrupted, essentially eliminating the earlier learned fear.

Owing to the findings of Nader et al (2000), there have been several studies exploring the nature of the blockade of reconsolidation. These studies point to both similarities and differences in the mechanisms underlying the initial consolidation of fear memories and reconsolidation (see Alberini, 2005: Dudai, 2006 for reviews). Importantly, these studies show that the blockade of reconsolidation is specific to the fear memory reactivated, leaving other memories intact (Doyere et al, 2007), and that fear memories do not return with passage of time, the alteration of contextual cues, or additional stress (Duvarci and Nader, 2004). Owing to the lack of return of fear, blocking reconsolidation provides an important advantage over other techniques that might be used to regulate emotion. If, as suggested, the memory trace is permanently altered, the inhibition of the fear memory through extinction, regulation, or adaptive action is not necessary.

Research on the blockade of the reconsolidation of fear memories in humans has been slow to emerge for a few reasons. One primary reason is that the initial findings showing the blockade of reconsolidation relied on the administration of protein synthesis inhibitors, which is not a viable technique in humans. Recently, however, two techniques that can be translated to humans have been identified. The first uses a beta-adrenergic antagonist, propranolol, which is safe to administer to humans, to block reconsolidation. Debiec and LeDoux (2004) showed that either intra-amygdala or systemic administration of propranolol immediately after reactivation of the CS blocked the reconsolidation of conditioned fear and prevented the return of fear. Interestingly, propranolol does not seem to block the initial consolidation of conditioned fear memories. Although the exact mechanism by which propranolol impacts reconsolidation is unknown, the effects of beta-adrenergic receptor blockade on reconsolidation may be due to the fact that these receptors modulate protein synthesis and thereby regulate long-term memory storage (Gelinas and Nguyen, 2005).

Given that propranolol is safe to use in human beings, the rodent study by Debiec and LeDoux (2004) provides a potential means for blocking reconsolidation in human beings. A recent finding by Kindt et al (2009) found that the administration of propranolol can block the return of fear in human participants. In this study, Kindt et al (2009) created a conditioned fear in the laboratory. After the fear memory was consolidated, the memory was reactivated by presenting the CS. Before reactivation, participants were given propranolol or placebo. Participants who received propranolol failed to show physiological evidence of conditioned fear when tested a day later and did not show any return of fear. Although these results are consistent with the findings of Debiec and LeDoux (2004) suggesting propranolol blocks reconsolidation, in the procedure of Kindt et al (2009), propranolol was given before 
reactivation. Owing to this, it is not clear whether propranolol in this study is effective by targeting a reconsolidation or reactivation mechanism. Theoretically, it is useful to specify which mechanism is preventing the return of fear, but practically it may be less important. If propranalol blocks the expression and return of fear memories through any mechanism, this suggests a potentially promising clinical intervention.

Earlier research has suggested that propranolol might be clinically useful if administered immediately after a traumatic event. It is suggested that propranolol might impair the initial consolidation of fear memories, and thus prevent the development of PTSD in the future (Pitman and Delahanty, 2005). However, this treatment is not applicable to someone who already suffers from PTSD. To explore whether the administration of propranolol during the reconsolidation period might be useful in the treatment of clinical disorders, Brunet et al (2008) tested whether it could prevent the return of older fear memories in patients suffering from PTSD. The patients were asked to describe their traumatic events to reactivate their traumatic memories. Immediately after reactivation, patients were given either propranolol or placebo. A week later during a script-driven memory task, physiological responses to these memories were assessed. The patients who received propranolol showed diminished physiological fear responses for most of the measures assessed. Although this initial clinical study had a small sample, moderate effects, and lacked some important control conditions, it provides preliminary evidence that propranolol could be an important tool in the treatment of anxiety disorders by targeting the reconsolidation process.

The studies examining means to block the reconsolidation of fear memories to date have primarily used pharmacological manipulations. However, reconsolidation is not a process that evolved via pharmacological manipulation in the service of treating clinical disorders. Rather reconsolidation is a natural memory process that allows older memories to be updated to incorporate new information each time they are retrieved. On account of this, it is possible that presenting specific new information during the reconsolidation window can provide another means to alter or block older fear memories. To explore whether the reconsolidation of fear memories can be influenced without pharmacological manipulation, Monfils et al (2009) developed a behavioral intervention aimed at blocking reconsolidation. The basic design of this study was similar to other rodent studies of reconsolidation, with one exception. Instead of administering a protein synthesis inhibitor during the reconsolidation window, Monfils et al (2009) presented an extinction learning session 10 min after CS reactivation. They found that rats that were given extinction training during the reconsolidation window failed to show any return of fear with the passage of time or after additional stress. This was in contrast to two control groups: (1) rats who received extinction training without reactivation and (2) those who received extinction training after reactivation, but outside the reconsolidation window (ie $6 \mathrm{~h}$ ). Both control groups showed evidence for the return of fear, consistent with research on extinction.

As outlined above, extinction training usually results in two competing memory traces, a CS-US trace that competes for expression with a CS-noUS trace. As both traces exist, fear can return with standard extinction training, as different circumstances favor the expression of one trace over another (Bouton, 2004). However, the study by Monfils et al (2009) suggests that extinction training during the reconsolidation window results in an alternative memory representation. When extinction learning occurs during the time period in which the original CS-US trace is labile, this original trace may be significantly altered to incorporate the CS-noUS learning before re-storage. The result is either an alternative or combined memory trace representing the significance of the CS. Importantly, this new memory trace does not support the return of fear.

By highlighting the adaptive function of reconsolidation in the absence of pharmacological manipulations, the results of Monfils et al (2009) suggest that reconsolidation may have a larger function in the regulation of emotion than was earlier appreciated. If we have the ability to alter emotional memories with every retrieval, we can potentially take advantage of our knowledge of reconsolidation mechanisms to develop specific behavioral manipulations that will change the expression of fear memories. This finding leads to several interesting possibilities that need be explored in future studies. For instance, can this be extended to humans (see Schiller et al, 2008), is it specific to targeted fear memories, and how will it extend to more complex memory traces, such as those representing traumatic emotional events outside the laboratory? Although there are several unanswered questions, the notion that we can use our knowledge of the natural reconsolidation process to change memory provides exciting possibilities for the development of novel, non-invasive treatments for psychological disorders.

\section{CONCLUSIONS AND FUTURE RESEARCH DIRECTIONS}

Research in both animal models and humans has enabled the development of an initial model of the neurocircuitry supporting the regulation of conditioned fear. Figure 1 outlines the network of brain regions hypothesized to support fear regulation through the four methods discussed in this review-extinction, cognitive regulation, active coping, and reconsolidation. Much of what we know about the neural mechanisms of fear regulation stems from research in animal models. Recent research in humans suggests that fear extinction is highly phylogenetically conserved, relying on the same neural substrates as other species. However, humans are also able to use cognitive strategies to regulate the expression of conditioned fear. Despite the fact that cognitive techniques may be deployed 


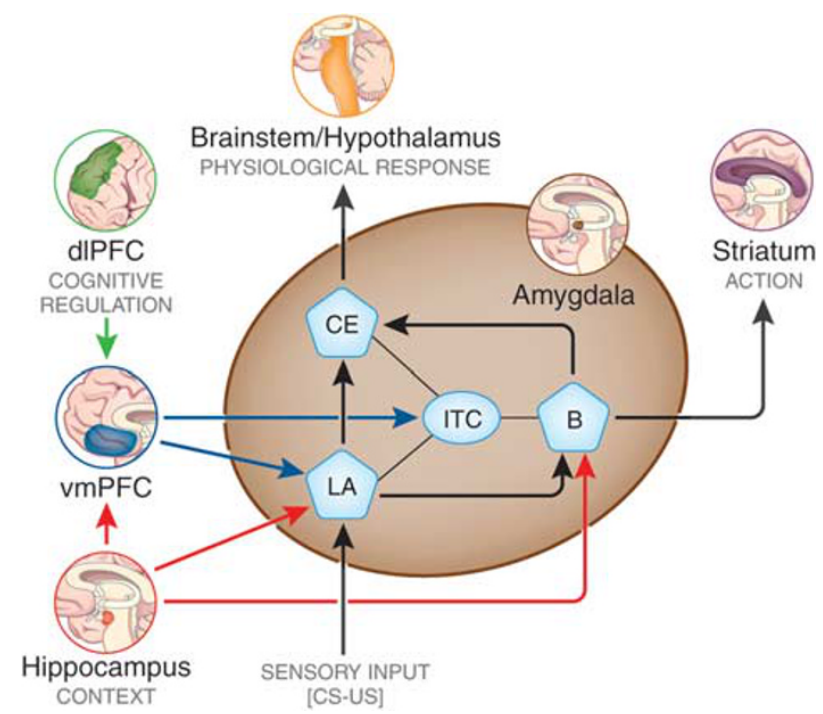

Figure 1. Model for the neurocircuitry of fear regulation in humans through extinction, cognitive regulation, active coping, and reconsolidation. A network of structures including the amygdala, hippocampus, VmPFC, dIPFC, and the striatum are involved in the regulation of conditioned fear expression. The lateral nucleus ( $\mathrm{LA})$ of the amygdala receives afferent sensory input and is the site of CS-US plasticity during fear conditioning. The LA projects to the central nucleus (CE), which has outputs to regions that control the expression of the CR. Projections from the hippocampus to the basal nucleus (B) of the amygdala process contextual information during conditioning, and may gate fear expression through the CE. During extinction learning and consolidation, inhibitory connections between the VMPFC and the intercalated (ITC) cell masses are established. During extinction recall, these connections inhibit fear expression through projections to the CE. Inhibitory connections between the $\mathrm{vmPFC}$ and the LA may also regulate fear expression during extinction recall through the CE. Contextual modulation of extinction expression is mediated by projections from the hippocampus to the vmPFC and/or LA. During cognitive regulation, the dorsolateral prefrontal cortex (dIPFC) regulates fear expression through projections to the vmPFC, which in turn inhibits amygdala activity. During active coping, information from the LA is routed not to the CE, which drives fear expression, but to the $\mathrm{B}$, which in turn projects to the striatum. The striatum is thought to reinforce instrumental action taken during escapefrom-fear or avoidance learning. Reconsolidation diminishes conditioned fear expression through alteration of the original CS-US association stored in the LA.

intentionally, unlike the relatively passive learning that takes place during extinction, the neural substrates supporting cognitive regulation appear to overlap in part with the regions involved in diminishing fear through extinction. Both extinction and cognitive regulation seem to use prefrontal inhibitory mechanisms to regulate amygdaladriven fear expression. Active coping and reconsolidation are two methods of fear regulation that are presently less well understood. Taking instrumental action to reduce exposure to fear-inducing contexts and stimuli is something we do regularly in our everyday lives. Although neuroscientific research is beginning to uncover the means by which such actions reduce fear expression, active coping seems to be an important means of controlling fear that has not yet been widely incorporated in clinical practice. Reconsolidation is an especially promising method of regulating fear, as its effects seem to be more robust, in that fear responses are less likely to reemerge. Given that stress impairs the inhibitory mechanisms involved in extinction and cognitive regulation, active coping and reconsolidation may provide avenues for regulating fear under conditions in which these methods are rendered ineffective. As future research improves our understanding of the neurocircuitry underlying these methods of emotion regulation and the contexts in which they are most effectively used, we will be better able to translate this research into new avenues of treatment for clinical disorders.

\section{DISCLOSURE}

Dr Phelps and Ms Hartley do not have any conflict of interests in relation to the material presented in this review article. Ms Hartley does not have any financial disclosures or income derived from organizations other than her employer (NYU) or government and private funding agencies. In addition to these funding sources, Dr Phelps is compensated for her role as an editor of a journal owned by the American Psychological Association.

\section{REFERENCES}

Alberini CM (2005). Mechanisms of memory stabilization: are consolidation and reconsolidation similar or distinct processes? Trends Neurosci 28: 51-56.

Allen LB, McHugh RK, Barlow DH (2008). Emotional disorders: a unified protocol. In Barlow DH (ed). Clinical Handbook of Psychological Disorders, 4th edn. Guilford Press: New York.

Amorapanth P, LeDoux JE, Nader K (2000). Different lateral amygdala outputs mediate reactions and actions elicited by a fear-arousing stimulus. Nat Neurosci 3: 74-79. Outlines differences in the neural circuitry mediating the expression of Pavlovian and Instrumental Fear learning.

Anderson KC, Insel TR (2006). The promise of extinction research for the prevention and treatment of anxiety disorders. Biol Psychiatry 60: 319-321.

Barbas $\mathrm{H}$ (2000). Connections underlying the synthesis of cognition, memory, and emotion in primate prefrontal cortices. Brain Res Bull 52: 319-330.

Barlow DH (ed). (2002). Anxiety and Its Disorders. The Nature and Treatment of Anxiety and Panic. Guilford Press: New York.

Barnes JL, Hill T, Langer M, Martinez M, Santos LR (2008). Helping behaviour and regard for others in capuchin monkeys (Cebus apella). Biol Lett 4: 638-640.

Beauregard M, Lévesque J, Bourgouin P (2001). Neural correlates of conscious self-regulation of emotion. J Neurosci 21: RC165.

Bouton ME (2004). Context and behavioral processes in extinction. Learn Mem 11: 485-494. Discusses the difference ways fear can return after extinction.

Bremner JD (2006). Traumatic stress: effects on the brain. Dialogues Clin Neurosci 8: $445-461$.

Brunet A, Orr SP, Tremblay J, Robertson K, Nader K, Pitman RK (2008). Effect of post-retrieval propranolol on psychophysiologic responding during subsequent script-driven traumatic imagery in post-traumatic stress disorder. J Psychiatr Res 46: 503-506. Preliminary evidence for the effectiveness of propranolol in blocking reconsolidation in a clinical population.

Bryant RA, Felmingham K, Kemp A, Das P, Hughes G, Peduto A et al (2007). Amygdala and ventral anterior cingulate activation predicts treatment response to cognitive behaviour therapy for post-traumatic stress disorder. Psychol Med 38: 556-561.

Burgos-Robles A, Vidal-Gonzalez I, Santini E, Quirk GJ (2007). Consolidation of fear extinction requires NMDA receptor-dependent bursting in the ventromedial prefrontal cortex. Neuron 53: 871-880.

Cain CK, LeDoux JE (2007). Escape from fear: a detailed behavioral analysis of two atypical responses reinforced by CS termination. J Exp Psychol Anim Behav Process 33: 451-463.

Cain CK, LeDoux JE (2008). Brain mechanisms of Pavlovian and instrumental aversive conditioning. In: Nutt DJ, Blanchard RJ, Blanchard DC, Griebel G (eds). Handbook of Anxiety and Fear. Elsevier Academic Press: Amsterdam. pp 103-125. 
Chhatwal JP, Myers KM, Ressler KJ, Davis M (2005). Regulation of gephyrin and GABAA receptor binding within the amygdala after fear acquisition and extinction. J Neurosci 25: 502-506.

Chhatwal JP, Stanek-Rattiner L, Davis M, Ressler KJ (2006). Amygdala BDNF signaling is required for consolidation but not encoding of extinction. Nat Neurosci 9: 870-872.

Choi JS, LeDoux JE (2003). Lesions of the lateral/basal but not the central nucleus of the amygdala impair post-training performance of fear-induced 2-way active avoidance signaled by a conditioned stimulus. Soc Neurosci Abst 29: 623.5.

Corcoran KA, Desmond TJ, Frey KA, Maren S (2005). Hippocampal inactivation disrupts the acquisition and contextual encoding of fear extinction. $J$ Neurosci 25: 8978-8987. Explores the role of the hippocampus in extinction and its retention

Corcoran KA, Maren S (2001). Hippocampal inactivation disrupts contextual retrieval of fear memory after extinction. J Neurosci 21: 1720-1726.

Corcoran KA, Maren S (2004). Factors regulating the effects of hippocampal inactivation on renewal of conditional fear after extinction. Learn Mem 11: 598-603.

Corcoran KA, Quirk GJ (2007). Recalling safety: cooperative functions of the ventromedial prefrontal cortex and the hippocampus in extinction. CNS Spectr 12: $200-206$

Debiec J, LeDoux JE (2004). Disruption of reconsolidation but not consolidation of auditory fear conditioning by noradrenergic blockade in the amygdala. Neurosci 129: 267-272. Demonstrates the effectiveness of propranolol in blocking reconsolidation.

Delgado MR, Jou RL, LeDoux JE, Phelps EA (2009). Avoiding negative outcomes: tracking the mechanisms of avoidance learning in humans during fear conditioning. Frontiers Behav Neurosci (in press).

Delgado MR, Nearing KI, LeDoux JE, Phelps EA (2008). Neural circuitry underlying the regulation of conditioned fear and its relation to extinction. Neuron 59: 829-838. Examines the overlap in the neural mechanisms of extinction and emotion regulation.

Doyère V, Debiec J, Monfils MH, Schafe GE, LeDoux JE (2007). Synapse-specific reconsolidation of distinct fear memories in the lateral amygdala. Nat Neurosci 4: 414-416.

Dudai $Y$ (2006). Reconsolidation: the advantage of being refocused. Curr Opin Neurobiol 16: 174-178.

Duvarci S, Nader K (2004). Characterization of fear memory reconsolidation. J Neurosci 24: 9269-9275.

Fanselow MS (2000). Contextual fear, gestalt memories, and the hippocampus. Behav Brain Res 110: 73-81.

Foa EB (2006). Psychosocial therapy for posttraumatic stress disorder. J Clin Psychiatry 67: 40-45.

Funayama ES, Grillon C, Davis M, Phelps EA (2001). A double dissociation in the affective modulation of startle in humans: effects of unilateral temporal lobectomy. J Cogn Neurosci 13: 721-729.

Gabriel M, Burhans L, Kashef A (2003). Consideration of a unified model of amygdalar associative functions. Ann NY Acad Sci 985: 206-217.

Garakani A, Mathew SJ, Charney DS (2006). Neurobiology of anxiety disorders and implications for treatment. Mt Sinai J Med 73: 941-949.

Gelinas JN, Nguyen PV (2005). Beta-adrenergic receptor activation facilitates induction of a protein synthesis-dependent late phase of long-term potentiation. J Neurosci 25: 3294-3303

Gilbertson MW, Shenton ME, Ciszewski A, Kasai K, Lasko NB, Orr SP et al (2002). Smaller hippocampal volume predicts pathologic vulnerability to psychological trauma. Nat Neurosci 5: 1242-1247.

Gottfried JA, Dolan RJ (2004). Human orbitofrontal cortex mediates extinction learning while accessing conditioned representations of value. Nat Neurosci 7: 1144-1152.

Gross JJ (1998). The emerging field of emotion regulation: an integrative review. Rev Gen Psychol 2: 271-299.

Gross JJ, Thompson RA (2007). Emotion regulation: conceptual foundations. In: Gross JJ (ed). Handbook of Emotion Regulation. Guilford Press: New York. pp 3-24.

Herry C, Trifilieff P, Micheau J, Luthi A, Mons N (2006). Extinction of auditory fear conditioning requires MAPK/ERK activation in the basolateral amygdala. Eur $J$ Neurosci 24: 261-269.

Hobin JA, Ji J, Maren S (2006). Ventral hippocampal muscimol disrupts contextspecific fear memory retrieval after extinction in rats. Hippocampus 16: 174-182.

Hugues S, Chessel A, Lena I, Marsault R, Garcia R (2006). Prefrontal infusion of PD098059 immediately after fear extinction training blocks extinction-associated prefrontal synaptic plasticity and decreases prefrontal ERK2 phosphorylation. Synapse 60: 280-287.

Ji J, Maren S (2005). Electrolytic lesions of the dorsal hippocampus disrupt renewal of conditional fear after extinction. Learn Mem 12: 270-276.

Kalisch R, Korenfeld E, Stephan KE, Weiskopf N, Seymour B, Dolan RJ (2006). Context-dependent human extinction memory is mediated by a ventromedial prefrontal and hippocampal network. J Neurosci 26: 9503-9511.
Kim H, Somerville LH, Johnstone T, Alexander AL, Whalen PJ (2003). Inverse amygdala and medial prefrontal cortex responses to surprised faces. Neuroreport 14: 2317-2322

Kim J, Lee S, Park H, Song B, Hong I, Geum D et al (2007). Blockade of amygdala metabotropic glutamate receptor subtype 1 impairs fear extinction. Biochem Biophys Res Commun 355: 188-193.

Kindt M, Soeter M, Vervliet B (2009). Beyond extinction: erasing human fear responses and preventing the return of fear. Nat Neurosci 12: 256-258. Demonstrates the use of proproanolol to disrupt the return of fear in humans.

Knight DC, Smith CN, Cheng DT, Stein EA, Helmstetter FJ (2004). Amygdala and hippocampal activity during acquisition and extinction of human fear conditioning. Cogn Affect Behav Neurosci 4: 317-325.

LaBar KS, Gatenby JC, Gore JC, LeDoux JE, Phelps EA (1998). Human amygdala activation during conditioned fear acquisition and extinction: a mixed-trial fMRI study. Neuron 20: 937-945.

LaBar KS, LeDoux JE, Spencer DD, Phelps EA (1995). Impaired fear conditioning following unilateral temporal lobectomy in humans. J Neurosci 15: 6846-6855.

LaBar KS, Phelps EA (2005). Reinstatement of conditioned fear in humans is context dependent and impaired in amnesia. Behav Neurosci 119: 677-686.

Lanius RA, Williamson PC, Densmore M, Boksman K, Gupta MA, Neufeld RW et al (2001). Neural correlates of traumatic memories in posttraumatic stress disorder: a functional MRI investigation. Am J Psychiatry 158: 1920-1922.

LeDoux JE (2000). Emotion circuits in the brain. Annu Rev Neurosci 23: 155-184.

LeDoux JE, Gorman JM (2001). A call to action: overcoming anxiety through active coping. Am J Psychiatry 158: 1953-1955. Discusses the use of active coping to control fear.

Liberzon I, Martis B (2006). Neuroimaging studies of emotional responses in PTSD. Ann NY Acad Sci 1071: 87-109.

Lin CH, Yeh SH, Lu HY, Gean PW (2003). The similarities and diversities of signal pathways leading to consolidation of conditioning and consolidation of extinction of fear memory. J Neurosci 23: 8310-8317.

Lindauer RJ, Booji J, Habraken JBA, Uylings HB, Olff M, Carlier IV et al (2004). Cerebral blood flow changes during script driven imagery in police officers with posttraumatic stress disorder. Biol Psychiatry 56: 853-861.

Maren S (2001). Neurobiology of Pavlovian fear conditioning. Annu Rev Neurosci 24: 897-931.

Maren S, Hobin JA (2007). Hippocampal regulation of context-dependent neuronal activity in the lateral amygdala. Learn Mem 14: 318-324. Demonstrates a hippocampus/amygdala pathway for controlling fear.

Markram K, Lopez Fernandez MA, Abrous DN, Sandi C (2007). Amygdala upregulation of NCAM polysialylation induced by auditory fear conditioning is not required for memory formation, but plays a role in fear extinction. Neurobiol Learn Mem 87: 573-582. 389-394.

Mauss IB, Bunge SA, Gross JJ (2007). Automatic emotion regulation. Soc Pers Psychol Compass 1: 146-167.

McDonald AJ, Mascagni F, Guo L (1996). Projections of the medial and lateral prefrontal cortices to the amygdala: a Phaseolus vulgaris leucoagglutinin study in the rat. Neurosci 71: 55-75.

Milad MR, Quinn BT, Pitman RK, Orr SP, Fischl B, Rauch SL (2005). Thickness of ventromedial prefrontal cortex in humans is correlated with extinction memory. Proc Natl Acad Sci USA 102: 10706-10711. Shows how individual variability in brain morphology is linked to retention of extinction.

Milad MR, Quirk GJ (2002). Neurons in medial prefrontal cortex signal memory for fear extinction. Nature 420: 70-74. Examines the response pattern of vmPFC neurons in exticntion.

Milad MR, Vidal-Gonzalez I, Quirk GJ (2004). Electrical stimulation of medial prefrontal cortex reduces conditioned fear in a temporally specific manner. Behav Neurosci 118: 389-394.

Milad MR, Wright Cl, Orr SP, Pitman RK, Quirk GJ, Rauch SL (2007). Recall of fear extinction in humans activates the ventromedial prefrontal cortex and hippocampus in concert. Biol Psychiatry 62: 446-454.

Monfils MH, Cowansage KK, Klann E, LeDoux JE (2009). Extinction-reconsolidation boundaries: key to persistent attenuation of fear memories. Science 324: 951-955.

Morgan MA, Romanski LM, LeDoux JE (1993). Extinction of emotional learning: contribution of medial prefrontal cortex. Neurosci Lett 163: 109-113.

Morgenson GJ, Jones DL, Yim CY (1980). From motivation to action: functional interface between the limbic system and the motor system. Prog Neurobiol 14: 69-97.

Nader K, Schafe GE, Le Doux JE (2000). Fear memories require protein synthesis in the amygdala for reconsolidation after retrieval. Nature 406: 722-726. Demonstrates that the reconsolidation of conditioned is protein synthesis dependent.

Ochsner KN, Bunge SA, Gross JJ, Gabrieli JD (2002). Rethinking feelings: an FMRI study of the cognitive regulation of emotion. J Cogn Neurosci 14: 1215-1229. 
Ochsner KN, Gross JJ (2008). Cognitive emotion regulation: insights from social, cognitive and affective neuroscience. Cur Dir Psych Sci 17: 153-158.

Olsson A, Phelps EA (2007). Social learning of fear. Nat Neurosci 10: 1095-1102.

Orr SP, Metzger LJ, Lasko NB, Macklin ML, Peri T, Pitman RK (2000). De novo conditioning in trauma-exposed individuals with and without posttraumatic stress disorder. J Abnorm Psychol 109: 290-298.

Paré D, Quirk GJ, LeDoux JE (2004). New vistas on amygdala networks in conditioned fear. J Neurophysiol 92: 1-9.

Pezawas L, Meyer-Lindenberg A, Drabant EM, Verchinski BA, Munoz KE, Kolachana BS et al (2005). 5-HTTLPR polymorphism impacts human cingulate-amygdala interactions: a genetic susceptibility mechanism for depression. Nat Neurosci 8: 828-834.

Phelps EA, Delgado MR, Nearing Kl, LeDoux JE (2004). Extinction learning in humans: role of the amygdala and vmPFC. Neuron 43: 897-905. Examines the neural systems of fear extinction and its retention in humans.

Phelps EA, LeDoux JE (2005). Contributions of the amygdala to emotion processing: from animal models to human behavior. Neuron 48: 175-187.

Phelps EA, O'Connor KJ, Gatenby JC, Gore JC, Grillon C, Davis M (2001). Activation of the left amygdala to a cognitive representation of fear. Nat Neurosci 4: 437-441.

Pitman RK, Delahanty DL (2005). Conceptually driven pharmacologic approaches to acute trauma. CNS Spectr 10: 99-106.

Quirk GJ, Likhtik E, Pelletier JG, Pare D (2003). Stimulation of medial prefrontal cortex decreases the responsiveness of central amygdala output neurons. $J$ Neurosci 23: 8800-8807.

Quirk GJ, Mueller D (2008). Neural mechanisms of extinction learning and retrieval. Neuropsychopharmacology 33: 56-72.

Quirk GJ, Russo GK, Barron JL, Lebron K (2000). The role of ventromedial prefrontal cortex in the recovery of extinguished fear. J Neurosci 20 : 6225-6231.

Rangel A, Camerer C, Montague PR (2008). A framework for studying the neurobiology of value-based decision making. Nat Rev Neuro 9: 545-556.

Rauch SL, Shin LM, Phelps EA (2006). Neurocircuitry models of posttraumatic stress disorder and extinction: human neuroimaging research — past, present, and future. Biol Psychiatry 60: 376-382.

Repa JC, Muller J, Apergis J, Desrochers TM, Zhou Y, LeDoux JE (2001). Two different lateral amygdala cell populations contribute to the initiation and storage of memory. Nat Neurosci 4: 724-731.

Ressler KJ, Rothbaum BO, Tannenbaum L, Anderson P, Graap K, Zimand et al (2004). Cognitive enhancers as adjuncts to psychotherapy: use of D-cycloserine in phobic individuals to facilitate extinction of fear. Arch Gen Psychiatry 61:
1136-1144. Provides evidence that a cognitive enhancer that impacts extinction can enhance the effectiveness of exposure therapy.

Rosenkranz JA, Moore H, Grace AA (2003). The prefrontal cortex regulates lateral amygdala neuronal plasticity and responses to previously conditioned stimuli. J Neurosci 23: 11054-11064.

Rothbaum BO, Schwartz AC (2002). Exposure therapy for posttraumatic stress disorder. Am J Psychother 56: 59-75.

Santini E, Ge H, Ren K, Pena DO, Quirk GJ (2004). Consolidation of fear extinction requires protein synthesis in the medial prefrontal cortex. J Neurosci 24: 5704-5710.

Scherer KR (2005). What are emotions? And how can they be measured? Soc Sci Inf 44: 695-729.

Schiller D, Monfils MH, Johnson DC, Raio CM, LeDoux JE, Phelps EA (2008). Behavioral blockade of return of fear in humans. Soc Neurosci 34: 591.16.

Shin LM, Rauch SL, Pitman RK (2006). Amygdala, medial prefrontal cortex, and hippocampal function in PTSD. Ann NY Acad Sci 1071: 67-79.

Sotres-Bayon F, Bush DE, LeDoux JE (2007). Acquisition of fear extinction requires activation of NR2B-containing NMDA receptors in the lateral amygdala. Neuropsychopharmacology 32: 1929-1940.

Sotres-Bayon F, Cain CK, LeDoux JE (2006). Brain mechanisms of fear extinction: historical perspectives on the contribution of prefrontal cortex. Biol Psychiatry 60: 329-336.

Urry HL, van Reekum CM, Johnstone T, Kalin NH, Thurow ME, Schaefer HS et al (2006). Amygdala and ventromedial prefrontal cortex are inversely coupled during regulation of negative affect and predict the diurnal pattern of cortisol secretion among older adults. J Neurosci 26: 4415-4425.

van der Kolk B (2006). Clinical implications of neuroscience research in PTSD. Ann N Y Acad Sci 1071: 277-293.

Wager TD, Davidson ML, Hughes BL, Lindquist MA, Ochsner KN (2008). Prefrontalsubcortical pathways mediating successful emotion regulation. Neuron 59 1037-1050.

Walker DL, Ressler KJ, Lu KT, Davis M (2002). Facilitation of conditioned fear extinction by systemic administration or intra-amygdala infusions of D-cycloserine as assessed with fear-potentiated startle in rats. J Neurosci 22: 2343-2351.

Wellman CL, Izquierdo A, Garrett JE, Martin KP, Carroll J, Millstein R et al (2007). Impaired stress-coping and fear extinction and abnormal corticolimbic morphology in serotonin transporter knock-out mice. J Neurosci 27: 684-691.

Wilson A, Brooks DC, Bouton ME (1995). The role of the rat hippocampal system in several effects of context in extinction. Behav Neurosci 109: 828-836. 